\title{
VARIATIONAL MEASURE
}

\author{
BY \\ MAURICE SION(1)
}

Introduction. The concept of a set which is $\nu$-measurable for all $\nu$ in some class $M$ of measures is possibly due to J. D. Tamarkin, who communicated some of his ideas to A. P. Morse around 1936. It is the latter, however, who first studied such sets extensively and, in a Real Variables course at the University of California, Berkeley, had them play a role in measure theory traditionally assumed by Borel sets.

Let $\mathfrak{M}_{0}$ be the set of outer measures $\nu$ on the real line such that open sets are $\nu$-measurable. A. P. Morse suggested to the author the problem of determining if a set, which is $\nu$-measurable for all $\nu$ in $\mathfrak{T l}_{0}$, is mapped into a similar set by a continuous function of bounded variation. It is while attempting to solve this problem that the writer was led to introduce a measure in the domain of a function, which is related to a given measure in the range and gives some indication of the extent to which the function fluctuates. This we have called variational measure, and in this paper we study some of its properties. As an application of the theory we have developed here, we get conditions on the function $f$ and class $M$ of measures in order that $f$ map a set, which is $\nu$-measurable for all $\nu$ in $M$, into a set of the same kind.

The problem suggested by A. P. Morse, however, remains open.

The approach to measure theory in this paper is that of $C$. Caratheodory and we refer the reader to [3] and [7] for the general background material.

1. Notation.

1.1. Ex $(\cdots)$ will denote the set of all $x$ such that $(\cdots)$.

1.2. $\{x\}$ will denote the set consisting of the point $x$ only.

1.3. $\sigma F=\bigcup A \in F A$.

1.4. $F$ is disjointed if and only if $A \in F, B \in F, A \neq B$ imply $A B=0$.

1.5. $\operatorname{dmn} f$ and $\mathrm{rng} f$ will denote respectively the domain and range of $f$.

1.6. $* f A=\mathrm{E} y(y=f(x)$ for some $x \in A)$.

1.7. ${ }^{*} f A=\mathrm{E} x(f(x)=y$ for some $y \in A)$.

1.8. When dealing with set functions $\nu$, we shall use the notation.$\nu A$ to stand for $\nu(A)$.

1.9. $\omega$ will denote the set of all non-negative integers.

1.10. A non-negative integer is the set of all non-negative integers preced-

Presented to the Society, February 25, 1956; received by the editors March 10, 1956.

(1) This research was supported by the United States Air Force, through the Office of Scientific Research of the Air Research and Development Command. 
ing it, that is: if $n \in \omega$ then $m \in n$ if and only if $m \in \omega$ and $m<n$. Thus, the number 0 is also the empty set.

\section{Fundamental definitions.}

2.1. A measure $\nu$ on $A$ is a function on the family of all subsets of $A$ to the non-negative real numbers such that . $\nu 0=0$ and if $B$ is a sequence of subsets of $A, C \subset \cup_{n} \in \omega B_{n}$, then

$$
. \nu C \leqq \sum n \in \omega . \nu B_{n} .
$$

2.2. $B$ is $\nu$-measurable if and only if for every $T \in \mathrm{dmn} \nu$,

$$
. \nu T=. \nu(T B)+. \nu(T-B) .
$$

2.3. $\nu$ is an outer measure if and only if $\nu$ is a measure and for every $B \in \mathrm{dmn} \nu$ there is a $B^{\prime}$ such that $B C B^{\prime}, B^{\prime}$ is $\nu$-measurable, and $. \nu B=. \nu B^{\prime}$.

2.4. Given a family $F$ of sets, we define:

$$
P(F)=\mathbf{E} P(P \subset \dot{F}, P \text { is finite, } P \text { is disjointed, } \sigma P=\sigma F) \text {. }
$$

2.5. $P^{\prime}$ is a refinement of $P$ if and only if for every $\alpha^{\prime} \in P^{\prime}$ there is an $\alpha \in P$ such that $\alpha^{\prime} \subset \alpha$, and $\sigma P=\sigma P^{\prime}$.

2.6. If $F$ is such that for every $P^{\prime}$ and $P^{\prime \prime}$ in $P(F)$ there is a $P$ in $\odot(F)$ which is a refinement of both, if for every $A \in F$, there is a $P \in \mathcal{P}(F)$ with $A \in P$, if $f$ is a function on $\sigma F$, and if $\nu$ is a measure on $\operatorname{rng} f$, we define the variational measure $V(F, f, \nu)$ to be the function $\mu$ on the subsets of $\sigma F$ such that for every $A \subset \sigma F$ :

$$
. \mu A=\sup _{P \in \mathcal{P}^{(P)}} \sum \alpha \in P . \nu_{*} f(A \alpha) .
$$

If the variables $F, f, \nu$ do not satisfy the above conditions, we define $V(F, f, \nu)$ to be the function $\mu$ such that $. \mu 0=0$ and $. \mu A=\infty$ for $0 \neq A \subset \sigma F$.

\section{General properties of variational measure.}

3.1. Theorem. $V(F, f, \nu)$ is a measure.

Proof. Let $\mu=V(F, f, \nu), B$ a sequence of subsets of $\sigma F, A=\bigcup_{n} \in \omega B_{n}$, $P \in \mathcal{P}(F)$. Then if $\nu$ is a measure:

$$
\begin{aligned}
\sum \alpha \in P . \nu_{*} f(A \alpha) & \\
& =\sum \alpha \in P . \nu \cup_{n} \in \omega_{*} f\left(B_{n} \alpha\right) \leqq \sum \alpha \in P \quad \sum n \in \omega . \nu_{*} f\left(B_{n} \alpha\right) \\
& =\sum n \in \omega \quad \sum \alpha \in P . \nu_{*} f\left(B_{n} \alpha\right) \leqq \sum n \in \omega . \mu B_{n} .
\end{aligned}
$$

Hence:

$$
. \mu A \leqq \sum n \in \omega . \mu B_{n} .
$$

All the other conditions are easily checked.

3.2. Lemma. If $P^{\prime}$ is a refinement of $P, P$ is disjointed, $f$ is a function on $\sigma P, \nu$ a measure on rng $f, A \subset \sigma P$, then: 


$$
\sum \alpha \in P . \nu_{*} f(A \alpha) \leqq \sum \alpha^{\prime} \in P^{\prime} . \nu_{*} f\left(A \alpha^{\prime}\right) .
$$

3.3. Theorem. If $\mu=V(F, f, \nu), A \in F$, then $A$ is $\mu$-measurable.

Proof. Let $. \mu T<\infty$. Then $. \mu(T A)<\infty$ and $. \mu(T-A)<\infty$. Given $\epsilon>0$, in view of the condition on $F(2.6)$ and Lemma 3.2, we can choose $P \in \mathcal{P}(F)$ such that

(1) for every $\alpha \in P$, if $\alpha A \neq 0$, then $\alpha \subset A$,

(2) $. \mu(T A) \leqq \sum \alpha \in P . \nu_{*} f(T A \alpha)+\epsilon$,

Let

(3) $. \mu(T-A) \leqq \sum \alpha \in P . \nu_{*} f((T-A) \alpha)+\epsilon$.

$$
\begin{aligned}
& P_{1}=\mathrm{E} \alpha(\alpha \in P \text { and } \alpha \subset A), \\
& P_{2}=\mathbf{E} \alpha(\alpha \in P \text { and } \alpha A=0) .
\end{aligned}
$$

Notice that $P=P_{1} \cup P_{2}$, and:

$$
\begin{aligned}
. \mu(T A)+. \mu(T-A) & \leqq \sum \alpha \in P_{1} \cdot \nu_{*} f(T A \alpha)+\sum \alpha \in P_{2} \cdot \nu_{*} f((T-A) \alpha)+2 \epsilon \\
& =\sum \alpha \in P_{1} \cdot \nu_{*} f(T \alpha)+\sum \alpha \in P_{2} \cdot \nu_{*} f(T \alpha)+2 \epsilon \\
& =\sum \alpha \in P . \nu_{*} f(T \alpha)+2 \epsilon \leqq . \mu T+2 \epsilon .
\end{aligned}
$$

Letting $\epsilon \rightarrow 0$, since $\mu$ is a measure, we see that

$$
. \mu T=. \mu(T A)+. \mu(T-A) .
$$

If $. \mu T=\infty$, the above equality is trivial.

3.4. Lemma. If $\mu=V(F, f, \nu), . \nu_{*} f A$ is a real number, and $. \nu_{0}=0$, then

$$
. \nu_{*} f A \leqq . \mu A .
$$

4. Properties of $V(F, f, \nu)$ when $\nu$ is an outer measure.

4.1. Lемма. If $f$ is a function on $\sigma F, \nu$ is an outer measure on $\operatorname{rng} f$, $P \in \mathcal{P}(F), A \subset B \subset \sigma F, . \nu_{*} f B<\infty$, then

$$
\sum \alpha \in P . \nu_{*} f(A \alpha)-. \nu_{*} f A \leqq \sum \alpha \in P . \nu_{*} f(B \alpha)-. \nu_{*} f B .
$$

Proof. Let $A^{*}={ }_{*} f A, B^{*}={ }_{*} f B, A^{\prime}$ be a $\nu$-measurable set such that $A^{*} C A^{\prime}$ and $. \nu A^{*}=. \nu A^{\prime}$. Since $. \nu B^{*}=. \nu A^{*}+. \nu\left(B^{*}-A^{\prime}\right)$, we need only show:

$$
\sum \alpha \in P . \nu_{*} f(A \alpha)+. \nu\left(B^{*}-A^{\prime}\right) \leqq \sum \alpha \in P . \nu_{*} f(B \alpha) .
$$

We use induction. If $P$ has only one element, the statement reduces to $. \nu_{*} f A+. \nu\left(B^{*}-A^{\prime}\right) \leqq \nu_{*} f B$ and hence is trivially true. Assume then that the inequality holds whenever $P$ has $n$ elements, and suppose that $P$ has $n+1$ elements: $\alpha_{0}, \cdots, \alpha_{n}$. Let $A_{1}^{*}=\bigcup k \in n_{*} f\left(A \alpha_{k}\right), A_{2}{ }^{*}={ }_{*} f\left(A \alpha_{n}\right), B_{1}{ }^{*}=\bigcup k \in n_{*} f\left(B \alpha_{k}\right), B_{2}{ }^{*}={ }_{*} f\left(B \alpha_{n}\right)$. 
Note that: $A^{*}=A_{1}{ }^{*} \cup A_{2}{ }^{*}$ and $B^{*}=B_{1}{ }^{*} \cup B_{2}{ }^{*}$. Next choose $\nu$-measurable sets $A_{1}^{\prime}$ and $A_{2}^{\prime}$ such that: $A_{1}^{*} \subset A_{1}^{\prime} \subset A^{\prime}, . \nu A_{1}^{*}=. \nu A_{1}^{\prime}, A_{2}^{*} \subset A_{2}^{\prime} \subset A^{\prime}, . \nu A_{2}^{*}=. \nu A_{2}^{\prime}$.

Note that: $B^{*}-A^{\prime} C\left(B_{1}^{*}-A_{1}^{\prime}\right) \cup\left(B_{2}^{*}-A_{2}^{\prime}\right)$ and $. \nu A_{2}^{*}+. \nu\left(B_{2}^{*}-A_{2}^{\prime}\right)$ $=. \nu B_{2}^{*}$. We then have:

$$
\begin{aligned}
\sum \alpha \in P . \nu_{*} f & (A \alpha)+. \nu\left(B^{*}-A^{\prime}\right) \\
& \leqq \sum k \in n . \nu_{*} f\left(A \alpha_{k}\right)+. \nu A_{2}{ }^{*}+. \nu\left(B_{1}^{*}-A_{1}^{\prime}\right)+. \nu\left(B_{2}{ }^{*}-A_{2}^{\prime}\right) \\
& \leqq \sum k \in n . \nu_{*} f\left(B \alpha_{k}\right)+. \nu B_{2}^{*}=\sum \alpha \in P . \nu_{*} f(B \alpha) .
\end{aligned}
$$

4.2. Theorem. If $\mu=V(F, f, \nu), \nu$ is an outer measure, $P$ is a sequence of elements in $\odot(F)$ such that

$$
\lim _{n \rightarrow \infty} \sum \alpha \in P_{n} . \nu_{*} f(B \alpha)=. \mu B<\infty,
$$

then, for any $A \subset B$

$$
\lim _{n \rightarrow \infty} \sum \alpha \in P_{n} . \nu_{*} f(A \dot{\alpha})=. \mu A .
$$

Proof. Let $A \subset B$. Given $\delta>0$, let $2 \epsilon=\delta$ and choose $N$ so that whenever $N<n \in \omega$ we have

$$
. \mu B<\sum \alpha \in P_{n} . \nu_{*} f(B \alpha)+\epsilon .
$$

Take $Q \in \mathcal{P}(F)$, a refinement of $P_{n}$, such that

$$
. \mu A<\sum \alpha \in Q . \nu_{*} f(A \alpha)+\epsilon .
$$

For each $\alpha \in P_{n}$, let $S_{\alpha}=\mathbf{E} \alpha^{\prime}\left(\alpha^{\prime} \in Q\right.$ and $\left.\alpha^{\prime} \subset \alpha\right)$. Then, using 4.1:

$$
\begin{array}{rl}
. \mu A-\sum \alpha \in P_{n} . \nu_{*} & f(A \alpha) \\
& <\sum \alpha \in Q . \nu_{*} f(A \alpha)-\sum \alpha \in P_{n} . \nu_{*} f(A \alpha)+\epsilon \\
& =\sum \alpha \in P_{n}\left[\sum \alpha^{\prime} \in S_{\alpha} \cdot \nu_{*} f\left(A \alpha^{\prime}\right)-. \nu_{*} f(A \alpha)\right]+\epsilon \\
& \leqq \sum \alpha \in P_{n}\left[\sum \alpha^{\prime} \in S_{\alpha} . \nu_{*} f\left(B \alpha^{\prime}\right)-. \nu_{*} f(B \alpha)\right]+\epsilon \\
& \leqq \sum \alpha \in Q . \nu_{*} f(B \alpha)-\sum \alpha \in P_{n} \cdot \nu_{*} f(B \alpha)+\epsilon<2 \epsilon=\delta .
\end{array}
$$

4.3. Lemma. If $P \in \mathcal{P}(F), f$ is a function on $\sigma F, \nu$ an outer measure on rng $f, B \subset \sigma F$, then there exists $a C \subset B$ such that:

$$
. \nu_{*} f(B \alpha)=. \nu_{*} f(C \alpha)+. \nu_{*} f((B-C) \alpha)
$$

for each $\alpha \in P$ and

$$
\sum \alpha \in P . \nu_{*} f(C \alpha)={ }_{\nu} f C=. \nu_{*} f B .
$$

Proof. Denote the elements of $P$ by $\alpha_{0}, \cdots, \alpha_{n-1}$. Let $B_{k}^{*}={ }_{*} f\left(B \alpha_{k}\right)$. Choose $\nu$-measurable sets $B_{k}{ }^{\prime}$ such that:

$B_{k}^{*} \subset B_{k}^{\prime}, . \nu B_{k}^{*}=. \nu B_{k}^{\prime}, . \nu \cup k \in r B_{k}^{*}=. \nu \cup k \in r B_{k}^{\prime} \quad$ for $r \in n+1$. 
We define

$$
C_{0}{ }^{*}=B_{0}{ }^{*} \text { and } C_{k}{ }^{*}=B_{k}{ }^{*}-\cup i \in k B_{i}^{\prime}
$$

We then let

$$
C_{k}=B \alpha_{k}^{*} f C_{k}^{*} \text { and } C=\bigcup k \in n C_{k} .
$$

We note first that the $C_{k}{ }^{*}$ are disjoint, ${ }_{*} f C_{k}=C_{k}^{*}$, and $C \alpha_{k}=C_{k}$. Also:

$$
C_{k}{ }^{*} f\left((B-C) \alpha_{k}\right)=0 \text { so that }{ }_{*} f\left((B-C) \alpha_{k}\right)=B_{k}{ }^{*} \cup i \in k B_{i}^{\prime} .
$$

Because of the $\nu$-measurability of the $B_{i}^{\prime}$, we conclude

$$
. \nu B_{k}^{*}=. \nu C_{k}^{*}+. \nu_{*} f\left((B-C) \alpha_{k}\right) .
$$

On the other hand we easily check by induction on $m$ that:

$$
. \nu \cup k \in m C_{k}{ }^{*}=\sum k \in m . \nu C_{k}{ }^{*}=. \nu \bigcup k \in m B_{k}{ }^{*} \quad \text { for } m \in n+1 .
$$

4.4. Theorem. If $\mu=V(F, f, \nu), \nu$ is an outer measure, $\mu B<\infty$, then there exists an $A \subset B$ such that:

$$
. \mu A=. \nu_{*} f A=. \nu_{*} f B .
$$

Proof. Let $P$ be a sequence of elements in $P(F)$ such that, for each $n \in \omega$, $P_{n+1}$ is a refinement of $P_{n}$ and

$$
. \mu B<\sum \alpha \in P_{n} . \nu_{*} f(B \alpha)+2^{-n} .
$$

By 4.3, we can choose a sequence $C$ such that, for all $n \in \omega, C_{n+1} \subset C_{n} \subset B$ and

$$
\begin{aligned}
\sum \alpha \in P_{n} . \nu_{*} f\left(C_{n} \alpha\right) & =. \nu_{*} f C_{n}=. \nu_{*} f(B), \\
. \nu_{*} f\left(C_{n} \alpha\right) & =. \nu_{*} f\left(C_{n+1} \alpha\right)+. \nu_{*} f\left(\left(C_{n}-C_{n+1}\right) \alpha\right) \quad \text { for } \alpha \in P_{n+1} .
\end{aligned}
$$

Let $A=\cap n \in \omega C_{n}$ and complete the proof in 5 steps.

$.1 . \mu A=($ by 4.2$)=\lim _{n \rightarrow \infty} \sum \alpha \in P_{n} . \nu_{*} f(A \alpha) \leqq \lim _{n \rightarrow \infty} \sum \alpha \in P_{n} . \nu_{*} f\left(C_{n} \alpha\right)$ $=. \nu * f B$

$.2 \sum \alpha \in P_{n+1} . \nu_{*} f\left(C_{n} \alpha\right)-\sum \alpha \in P_{n} . \nu_{*} f\left(C_{n} \alpha\right) \leqq 2^{-n}$.

Proof. For $\alpha \in P_{n}$, let $S_{\alpha}=E \alpha^{\prime}\left(\alpha^{\prime} \in P_{n+1}\right.$ and $\left.\alpha^{\prime} \subset \alpha\right)$. Then, using 4.1:

$$
\sum \alpha \in P_{n}\left[\sum \alpha^{\prime} \in S_{\alpha} \cdot \nu_{*} f\left(C_{n} \alpha \alpha^{\prime}\right)-. \nu_{*} f\left(C_{n} \alpha\right)\right]
$$

$$
\begin{aligned}
& \leqq \sum \alpha \in P_{n}\left[\sum \alpha^{\prime} \in S_{\alpha} \cdot \nu_{*} f\left(B \alpha \alpha^{\prime}\right)-. \nu_{*} f(B \alpha)\right] \\
& =\sum \alpha \in P_{n+1} \cdot \nu_{*} f(B \alpha)-\sum \alpha \in P_{n} \cdot \nu_{*} f(B \alpha) \leqq 2^{-n} .
\end{aligned}
$$

$.3 . \nu_{*} f\left(C_{n}-C_{n+1}\right) \leqq 2^{-n}$.

Proof.

$$
\begin{aligned}
. \nu_{*} f\left(C_{n}-C_{n+1}\right) & \leqq \sum \alpha \in P_{n+1} . \nu_{*} f\left(\left(C_{n}-C_{n+1}\right) \alpha\right) \\
& =\sum \alpha \in P_{n+1} . \nu_{*} f\left(C_{n} \alpha\right)-\sum \alpha \in P_{n+1} . \nu_{*} f\left(C_{n+1} \alpha\right) \\
& =\sum \alpha \in P_{n+1} . \nu_{*} f\left(C_{n} \alpha\right)-\sum \alpha \in P_{n} . \nu_{*} f\left(C_{n} \alpha\right) \leqq 2^{-n} .
\end{aligned}
$$


$.4 . \mu\left(C_{n}-C_{n+1}\right) \leqq . \nu_{*} f\left(C_{n}-C_{n+1}\right)+2^{-n} \leqq 2^{-n+1}$.

Proof. Let $S_{\alpha}^{(m)}=\mathbf{E} \alpha^{\prime}\left(\alpha^{\prime} \in P_{n+m}\right.$ and $\left.\alpha^{\prime} \subset \alpha\right)$. Then

$$
\sum \alpha \in P_{n+m} \cdot \nu_{*} f\left(\left(C_{n}-C_{n+1}\right) \alpha\right)
$$

$$
\begin{aligned}
& =\sum \alpha \in P_{n} \sum \alpha^{\prime} \in S_{\alpha}^{(m)} . \nu_{*} f\left(\left(C_{n}-C_{n+1}\right) \alpha^{\prime}\right) \leqq(\text { by } 4.1) \\
& \leqq \sum \alpha \in P_{n}\left[. \nu_{*} f\left(\left(C_{n}-C_{n+1}\right) \alpha\right)+\sum \alpha^{\prime} \in S_{\alpha}^{(m)} . \nu_{*} f\left(B \alpha^{\prime}\right)-. \nu_{*} f(B \alpha)\right] \\
& =. \nu_{*} f\left(C_{n}-C_{n+1}\right)+\sum \alpha \in P_{n+m} . \nu_{*} f(B \alpha)-\sum \alpha \in P_{n} . \nu_{*} f(B \alpha) \\
& \leqq . \nu_{*} f\left(C_{n}-C_{n+1}\right)+2^{-n} .
\end{aligned}
$$

Using 4.2 and letting $m \rightarrow \infty$ we have the desired conclusion.

$$
.5 . \nu_{*} f B=. \mu A \text {. }
$$

Proof. . $\mu A \geqq . \mu C_{n}-\sum m \in \omega \cdot \mu\left(C_{n+m}-C_{n+m+1}\right) \geqq \nu_{*} f B-\sum m \in \omega 2^{-n-m+1}$ $\geqq \cdot \nu_{*} f B-2^{-n+2}$.

Letting $n \rightarrow \infty$ and using Step 1, we have the desired result.

4.5. Theorem. If $\mu=V(F, f, \nu), \nu$ is an outer measure, $A$ is $\nu$-measurable, then ${ }^{*} f A$ is $\mu$-measurable.

Proof. Let . $\mu T<\infty$. Choose a sequence $P$ of elements in $P(F)$ such that $. \mu T=\lim _{n \rightarrow \infty} \sum \alpha \in P_{n} . \nu_{*} f(T \alpha)$. Letting $B={ }^{*} f A$, we see by 4.2 that:

$$
\begin{aligned}
. \mu(T B)+. \mu(T-B) & =\lim _{n \rightarrow \infty} \sum \alpha \in P_{n}\left[. \nu_{*} f(T B \alpha)+. \nu_{*} f((T-B) \alpha)\right] \\
& =\lim _{n \rightarrow \infty} \sum \alpha \in P_{n} . \nu_{*} f(T \alpha)=. \mu T .
\end{aligned}
$$

The next to last equality is due to the $\nu$-measurability of $A={ }_{*} f B$ and the fact that $* f(T B \alpha) \subset A$ and $* f((T-\dot{B}) \alpha) C_{*} f(T \alpha)-A$.

4.6. TheOREM. If $\mu=V(F, f, \nu), \nu$ is an outer measure, $\mu A=. \nu_{*} f A<\infty$, * $f A$ is $\nu$-measurable, $\alpha \in F$, then $* f(A \alpha)$ is $\nu$-measurable.

Proof. Choose a $\nu$-measurable set $B$ such that:

$$
{ }_{*} f(A \alpha) \subset B \subset{ }_{*} f A \text { and } . \nu_{*} f(A \alpha)=. \nu B .
$$

Since $. \mu A=. \nu_{*} f A$, we must have: $. \nu_{*} f A=. \nu_{*} f(A \alpha)+. \nu_{*} f(A-\alpha)$. Since $B$ is $\nu$-measurable, we also have:

$$
\begin{aligned}
. \nu_{*} f(A-\alpha) & =. \nu(* f(A-\alpha) B)+. \nu\left({ }_{*} f(A-\alpha)-B\right), \\
. \nu_{*} f A & \left.=. \nu B+. \nu(* f A-B)=. \nu_{*} f(A \alpha)+. \nu{ }_{*} f A-B\right) .
\end{aligned}
$$

Now, $* f(A-\alpha)-B=* f A-B$. Hence, comparing the two expressions above for.$\nu_{*} f A$, we conclude that $. \nu\left({ }_{*} f(A-\alpha) B\right)=0$ and hence.$\nu\left(B-{ }_{*} f(A \alpha)\right)$ $\leqq . \nu(* f(A-\alpha) B)=0$.

5. Properties of $V(F, f, \nu)$ when $F$ is fine.

5.1. Definition. $P$ is a mesh of $F$ if and only if $P$ is a sequence of elements 
in $P(F)$ such that: $P_{n+1}$ is a refinement of $P_{n}$ and, for every $x \in \sigma F, y \in \sigma F$, $x \neq y$, there exists an $n \in \omega, \alpha \in P_{n}, \beta \in P_{n}, \alpha \beta=0$ such that $x \in \alpha$ and $y \in \beta$.

5.2. Definition. $F$ is fine if and only if: $F \neq 0$; for every $A \in F$ there is a $P \in \mathcal{P}(F)$ with $A \in P$; for every $P^{\prime}$ and $P^{\prime \prime}$ in $P(F)$ there is a mesh $P$ of $F$ such that for every $n \in \omega, P_{n}$ is a refinement of both $P^{\prime}$ and $P^{\prime \prime}$.

5.3. Definition. $N(f, A, y)$ denotes the number of points in $A^{*} f\{y\}$ if this set is finite and $\infty$ otherwise.

5.4. Definition. $\operatorname{Cr}(A, y)$ is 1 if $y \in A$ and 0 otherwise.

5.5. Lemma. If $f$ is a function on $\sigma F, P$ is a mesh of $F$, then

$$
N(f, A, y)=\sup _{n \in \omega} \sum \alpha \in P_{n} \operatorname{Cr}\left({ }_{*} f(A \alpha), y\right) .
$$

Proof. Clearly, for every $n \in \omega$ :

$$
\sum \alpha \in P_{n} \operatorname{Cr}\left({ }_{*} f(A \alpha), y\right) \leqq N(f, A, y) .
$$

Let $m \leqq N(f, A, y), m \in \omega$. Take $m$ distinct points $x_{1}, \cdots, x_{m}$ in $A$ with $f\left(x_{i}\right)=y$. Then choose $n \in \omega$ such that no $\alpha$ in $P_{n}$ contains more than one of these points. We must therefore have

$$
m \leqq \sum \alpha \in P_{n} \operatorname{Cr}(* f(A \alpha), y) .
$$

5.6. ThEOREM. If $\mu=V(F, f, \nu), F$ is fine, $* f(A \alpha)$ is $\nu$-measurable for every $\alpha \in F$, then . $\mu A=\int N(f, A, y) d \nu y$.

Proof. Let $P$ be a mesh of $F$ such that

$$
. \mu A=\lim _{n \rightarrow \infty} \sum \alpha \in P_{n} . \nu_{*} f(A \alpha) .
$$

Since ${ }_{*} f(A \alpha)$ is $\nu$-measurable for every $\alpha \in \dot{P}_{n}$, we see from 5.5 that $N(f, A, y)$ is $\nu$-measurable in $y$ and:

$$
\begin{aligned}
. \mu A & =\lim _{n \rightarrow \infty} \sum \alpha \in P_{n} \int \operatorname{Cr}\left({ }_{*} f(A \alpha), y\right) d \nu y . \\
& =\int \lim _{n \rightarrow \infty} \sum \alpha \in P_{n} \operatorname{Cr}\left({ }_{*} f(A \alpha), y\right) d \nu y=\int N(f, A, y) d \nu y .
\end{aligned}
$$

5.7. Corollary. If $\mu=V(F, f, \nu), F$ is fine, $\nu$ is an outer measure, . $\mu A$ $=. \nu_{*} f A<\infty, * f A$ is $\nu$-measurable, then $N(f, A, y) \leqq 1$ for $\nu$-almost all $y$.

Proof. Follows immediately from 4.6 and 5.6.

5.8. TheOREM. If $\mu=V(F, f, \nu), F$ is fine, $\nu$ is an outer measure, . $\mu A$ $=. \nu_{*} f A<\infty, * f A$ is $\nu$-measurable, then $A$ is $\mu$-measurable.

Proof. Let $P$ be a mesh of $F$. For each $n \in \omega$ and $\alpha \in P_{n}$, let $C_{\alpha}=\alpha^{*} f_{*} f(A \alpha)$ and $D_{n}=\cup_{\alpha} \in P_{n} C_{\alpha}$. 
Since ${ }_{*} f(A \alpha)$ is $\nu$-measurable, then by 3.3 and 4.5 we see that the $C_{\alpha}$ and hence $D_{n}$ are $\mu$-measurable. Also: $D_{n} \alpha=C_{\alpha},{ }_{*} f\left(D_{n} \alpha\right)={ }_{*} f(A \alpha)$, and $A \subset D_{n}$.

Let $B=\bigcap n \in \omega D_{n}$. Then $B$ is $\mu$-measurable, $A \subset B$, and for each $\alpha \in P_{n}$ : ${ }_{*} f(A \alpha) \subset_{*} f(B \alpha) \subset_{*} f\left(D_{n} \alpha\right)={ }_{*} f(A \alpha)$. Thus, by 5.5 and $5.7, N(f, B, y)$ $=N(f, A, y) \leqq 1$ for $\nu$-almost all $y$. Hence $N(f, B-A, y)=0$ for $\nu$-almost all $y$, so that $. \nu_{*} f(B-A)=0$. Therefore $. \mu(B-A)=0$ and $A$ is $\mu$-measurable.

REMARK. We are unable to prove, in general, that $\mu=V(F, f, \nu)$ is an outer measure when $F$ is fine and $\nu$ is an outer measure. Since we are interested in the converse of Theorem 5.8, we use the same conditions to prove both results. It may be of interest to note, however, that the two are not naturally related. For example, if $F$ is the family of all intervals on the real line and $\nu$ is an outer measure, then $\mu$ is also an outer measure. On the other hand, it is quite clear that the converse of 5.8 is not true for all functions $f$ and all outer measures $\nu$ on $\operatorname{rng} f$.

6. Properties of $V(F, f, \nu)$ when $\sigma F$ is a topological space. Throughout this section we assume a fixed topology for $\sigma F$. All the topological concepts refer to this topology.

6.1. Definition. For $P$ a mesh of $F$, we denote by $S q(P)$ the set of sequences $\alpha$ such that $0 \neq \alpha_{n+1} \subset \alpha_{n} \in P_{n}$, for every $n \in \omega$.

6.2. Definition. $P$ is a topological mesh of $F$ if and only if: $P$ is a mesh of $F$; for every $x \in \sigma F$ and neighborhood $U$ of $x$, there is an $n \in \omega$ and $\alpha \in P_{n}$ such that $x \in \alpha \subset U$.

6.3. Definition. $F$ is almost complete if and only if: $F$ is fine; there exists a topological mesh $P$ of $F ; \mathbf{E} \alpha\left(\alpha \in S q(P), \cap n \in \omega \alpha_{n}=0\right)$ is countable for all topological meshes $P$ of $F$.

6.4. Definition. $\mathfrak{T}(F, f)=\mathbf{E} \nu$ ( $\nu$ is an outer measure on $\operatorname{rng} f ; \operatorname{dmn} f \subset \sigma F$; ${ }_{*}^{*} \alpha \alpha$ is $\nu$-measurable for all $\alpha \in F ;{ }^{*}\{y\}$ is closed for $\nu$-almost all $y$ ).

6.5. Lemia. If $P$ is a mesh of $F$, for every $n \in \omega: 0 \neq A_{n+1} \subset A_{n} \subset \sigma F$, then for some $\alpha \in S q(P)$ and every $n \in \omega: A_{n} \alpha_{n} \neq 0$.

Proof. We define $\alpha$ by recursion. Since $P_{n}$ is finite and $\sigma P_{n}=\sigma F$ for every $n \in \omega$, we can choose $\alpha_{0} \in P_{0}$ so that $\alpha_{0} A_{k} \neq 0$ for an infinite number of, and hence all, $k \in \omega$. Having chosen $\alpha_{n}$ so that $\alpha_{n} A_{k} \neq 0$ for all $k \in \omega$, we take $\alpha_{n+1} \in P_{n+1}$ so that $\alpha_{n+1} \subset \alpha_{n}$ and $\alpha_{n+1} A_{k} \neq 0$ for all $k \in \omega$.

6.6. Lemma. If $F$ is almost complete, $A \in F, P$ is a topological mesh of $F$, and $S=\mathbf{E} \alpha\left(\alpha \in S q(P) ; A \alpha_{n} \neq 0\right.$ for all $\left.n \in \omega ; \cap n \in \omega\left(A \alpha_{n}\right)=0\right)$, then $S$ is countable.

Proof. Let $A \in Q \in P(F)$ and choose $P^{\prime}$ a mesh of $F$ such that, for every $n \in \omega, P_{n}^{\prime}$ is a refinement of both $Q$ and $P_{n}$. Let $S^{\prime}=\mathrm{E} \alpha^{\prime}\left(\alpha^{\prime} \in S q\left(P^{\prime}\right) ; A \alpha_{n}^{\prime} \neq 0\right.$ for all $\left.n \in \omega ; \cap n \in \omega\left(A \alpha_{n}^{\prime}\right)=0\right)$. Since, for $\alpha_{n}^{\prime} \in P_{n}^{\prime}, A \alpha_{n}^{\prime} \neq 0$ implies $A \alpha_{n}^{\prime}$ $=\alpha_{n}^{\prime}$, we see that $S^{\prime}$ is countable. By 6.5 , for each $\alpha \in S$ there exists $\alpha^{\prime} \in S^{\prime}$ such that $\alpha_{n}^{\prime} \alpha_{n} \neq 0$ and hence $\alpha_{n}^{\prime} \subset \alpha_{n}$ for all $n \in \omega$. This means that for two 
different $\alpha^{\prime}$ 's in $S$, the corresponding $\alpha^{\prime \prime}$ s in $S^{\prime}$ are also different. Thus $S$ is also countable.

6.7. Lemma. If $B C_{*} f \alpha, A=\alpha^{*} f B, \alpha^{\prime} \subset \alpha$, then ${ }_{*} f\left(A \alpha^{\prime}\right)={ }_{*} f A_{*} f \alpha^{\prime}=B_{*} f \alpha^{\prime}$.

6.8. Lemma. If $F$ has a topological mesh $P, \nu \in \mathfrak{T}(F, f), \mu=V(F, f, \nu)$, $x \in \sigma F$, then $\{x\}$ is $\mu$-measurable and $* f\{x\}$ is $\nu$-measurable.

Proof. Choose a sequence $\alpha$ so that for every $n \in \omega, \alpha_{n} \in P_{n}$ and $\{x\}$ $=\bigcap n \in \omega \alpha_{n}$. By 3.3 the $\alpha_{n}$ are $\mu$-measurable and hence $\{x\}$ is $\mu$-measurable. Let $A=\bigcap n \in \omega * f \alpha_{n}$. Then $A$ is $\nu$-measurable. Let $y=f(x)$ and $A^{*}=\mathrm{E} z(z \in A$ and ${ }^{*}\{z\}$ is closed). We note that: . $\nu\left(A-A^{*}\right)=0$; if $z \in A^{*}$, then $x$ is in the closure of ${ }^{*}\{z\}$, hence $x \in{ }^{*}\{z\}$ and $y=z$. Thus, if $. \nu\{y\} \neq 0$, then $\{y\}=A^{*}$ and in either case $\{y\}$ is $\nu$-measurable.

6.9. Lemma. If $P$ is a mesh of $F, \alpha \in S q(P), \lim _{n \rightarrow \infty} \sum \alpha^{\prime} \in P_{n} . \nu_{*} f\left(A \alpha^{\prime}\right)$ $<\infty, \nu$ is an outer measure, and $. \nu\{y\}=0$ for all $y \in \in_{*} f A$, then $\lim _{n \rightarrow \infty} . \nu_{*} f\left(A \alpha_{n}\right)$ $=0$.

Proof. Let $B=\bigcap n \in \omega \alpha_{n}$. Then $B$ contains at most one point and . $\nu_{*} f(A B)$ $=0$. Since $* f\left(A \alpha_{n}\right)=\bigcup k \in \omega * f\left(A \alpha_{n}-\alpha_{n+k}\right) \cup_{*} f(A B)$, we must have

$$
\lim _{k \rightarrow \infty} . \nu_{*} f\left(A \alpha_{n}-\alpha_{n+k}\right)=. \nu_{*} f\left(A \alpha_{n}\right)
$$

for all $n \in \omega$. Now, given $\epsilon>0$ we can choose $n$ so large that for all $k \in \omega$ :

$$
. \nu_{*} f\left(A \alpha_{n}-\alpha_{n+k}\right)+. \nu_{*} f\left(A \alpha_{n+k}\right) \leqq . \nu_{*} f\left(A \alpha_{n}\right)+\epsilon .
$$

Hence: $\lim _{k \rightarrow \infty} \cdot \nu_{*} f\left(A \alpha_{n+k}\right) \leqq . \nu_{*} f\left(A \alpha_{n}\right)-\lim _{k \rightarrow \infty} \cdot \nu_{*} f\left(A \alpha_{n}-\alpha_{n+k}\right)+\epsilon=\epsilon$. Since $\epsilon$ is arbitrary we have the desired conclusion.

6.10. Theorem. If $F$ is almost complete, $\nu \in \mathfrak{T}(F, f), \mu=V(F, f, \nu), P$ is a topological mesh of $F$, then for any $A \subset \sigma F$ there is a $\mu$-measurable $A^{\prime}$ such that $A \subset A^{\prime}, * f A^{\prime}$ is $\nu$-measurable, and

$$
. \mu A=. \mu A^{\prime}=\lim _{n \rightarrow \infty} \sum \alpha \in P_{n} . \nu_{*} f\left(A^{\prime} \alpha\right)
$$

Proof. If $\lim _{n \rightarrow \infty} \sum \alpha \in P_{n} . \nu_{*} f(A \alpha)=\infty$, take $A^{\prime}=\sigma F$. Otherwise, set $A_{1}=\mathrm{E} x\left(x \in A\right.$ and $\left.. \nu_{*} f\{x\}>0\right)$. Then $A_{1}$ is countable and, by $6.8,{ }_{*} f A_{1}$ is $\nu$-measurable. Thus we need only prove the theorem for $A_{2}=A-A_{1}$. We define the sets $B_{\alpha}^{(n)}, C_{\alpha}^{(n)}, D_{n}$ by recursion on $n$. For $\alpha \in P_{0}$, let $B_{\alpha}^{(0)}$ be $\nu$-measurable, ${ }_{*} f\left(A_{2} \alpha\right) \subset B_{\alpha}^{(0)} C_{*} f \alpha$, and $. \nu_{*} f\left(A_{2} \alpha\right)=. \nu B_{\alpha}^{(0)}$. Then we set

$$
C_{\alpha}^{(0)}=\alpha^{*} f B_{\alpha}^{(0)} \text { and } D_{0}=\bigcup_{\alpha} \in P_{0} C_{\alpha}^{(0)} \text {. }
$$

Using 6.7, for $\alpha \in P_{n+1}$, let $B_{\alpha}^{(n+1)}$ be $\nu$-measurable, ${ }_{*} f\left(A_{2} \alpha\right) \subset B_{\alpha}^{(n+1)} C_{*} f\left(D_{n} \alpha\right)$, and $. \nu_{*} f\left(A_{2} \alpha\right)=. \nu B_{\alpha}^{(n+1)}$, then we set: 


$$
C_{\alpha}^{(n+1)}=\alpha^{*} f B_{\alpha}^{(n+1)} \text { and } D_{n+1}=\bigcup_{\alpha} \in P_{n+1} C_{\alpha}^{(n+1)} .
$$

Finally, let $A^{\prime}=n_{n} \in \omega D_{n}$. Clearly, $A_{2} \subset A^{\prime}, A^{\prime}$ is $\mu$-measurable since the $C_{\alpha}^{(n)}$ are $\mu$-measurable by 3.3 and 4.5 , and for $n \in \omega$ and $\alpha \in P_{n}$ :

$$
. \nu_{*} f\left(A_{2} \alpha\right) \leqq . \nu_{*} f\left(A^{\prime} \alpha\right) \leqq . \nu_{*} f\left(D_{n} \alpha\right)=. \nu B_{\alpha}^{(n)}=. \nu_{*} f\left(A_{2} \alpha\right)
$$

Now, we wish to show that $* f\left(A^{\prime} \beta\right)$ is $\nu$-measurable for any $\beta \in F$ and.$\mu A_{2}$ $=. \mu A^{\prime}$. To this end, we let $B^{\prime}=\cap n \in \omega_{*} f\left(D_{n} \beta\right), B^{*}=\mathrm{E} y\left(y \in B^{\prime}\right.$ and ${ }^{*} f\{y\}$ is closed), and, for $\alpha \in S q(P), G_{\alpha}=\mathbf{E} y\left(y \in B^{*}\right.$ and, for $\left.n \in \omega, \beta D_{n} \alpha_{n} *_{f}\{y\} \neq 0\right)$, and complete the proof in 7 steps.

Part 1. $B^{\prime}$ is $\nu$-measurable, . $\nu\left(B^{\prime}-B^{*}\right)=0$, and $B^{*}$ is $\nu$-measurable.

Proof. $\nu \in \mathfrak{M}(F, f)$ and $* f\left(D_{n} \beta\right)$ is $\nu$-measurable.

Part 2. $B^{*}=\bigcup_{\alpha} \in S q(P) G_{\alpha}$.

Proof. Clearly $G_{\alpha} \subset B^{*}$ for all $\alpha \in S q(P)$. If $y \in B^{*}$, then $D_{n} \beta^{*}\{y\} \neq 0$ for all $n \in \omega$ and hence by 6.5 there is an $\alpha \in S q(P)$ such that $\beta D_{n} \alpha_{n}{ }^{*}\{y\} \neq 0$, i.e., $y \in G_{\alpha}$.

Part 3. If $\alpha \in S q(P)$ and $\cap n \in \omega\left(\beta \alpha_{n}\right) \neq 0$, then $G_{\alpha} \subset_{*} f\left(A^{\prime} \beta\right)$.

Proof. Let $x \in \cap n \in \omega\left(\beta \alpha_{n}\right)$ and $y \in G_{\alpha}$. Then $x$ is in the closure of ${ }^{*}\{y\}$, hence $x \in{ }^{*} f\{y\}$. Since $D_{n} \alpha_{n}{ }^{*}\left\{\{y\} \neq 0\right.$ we have $\alpha_{n}{ }^{*}\{y\} \subset C_{\alpha_{n}}^{(n)} \subset D_{n}$. Thus $x \in D_{n}$ for all $n \in \omega$ and therefore $x \in A^{\prime} \beta^{*} f\{y\}$, so that $y \in{ }_{*} f\left(A^{\prime} \beta\right)$.

Part 4. E $\alpha\left(\alpha \in S q(P) ; G_{\alpha} \neq 0 ; \cap n \in \omega\left(\beta \alpha_{n}\right)=0\right)$ is countable.

Proof. Follows immediately from 6.6.

Part 5. $\nu G_{\alpha}=0$ for all $\alpha \in S q(P)$.

Proof. $G_{\alpha} C_{*} f\left(D_{n} \alpha_{n}\right)$ for all $n \in \omega$, and hence by 6.9

$$
. \nu G_{\alpha} \leqq \lim _{n \rightarrow \infty} . \nu_{*} f\left(D_{n} \alpha_{n}\right)=\lim _{n \rightarrow \infty} . \nu_{*} f\left(A_{2} \alpha_{n}\right)=0 .
$$

Part 6. . $\nu\left(B^{*}-{ }_{*} f\left(A^{\prime} \beta\right)\right)=0$ and ${ }_{*} f\left(A^{\prime} \beta\right)$ is $\nu$-measurable.

Proof. Clearly $* f\left(A^{\prime} \beta\right) \subset B^{*} \cup\left(B^{\prime}-B^{*}\right)$. Let $S$ be the countable set of Part 4. Then, in view of Parts 2 and 3:

$$
B^{*}-{ }_{*} f\left(A^{\prime} \beta\right) \subset \cup_{\alpha} \in S G_{\alpha}
$$

and by Part 5:

$$
. \nu\left(B^{*}-{ }_{*} f\left(A^{\prime} \beta\right)\right) \leqq \sum \alpha \in S . \nu G_{\alpha}=0 .
$$

Part 7. $\mu A_{2}=. \mu A^{\prime}=\lim _{n \rightarrow \infty} \sum \alpha \in P_{n} . \nu_{*} f\left(A^{\prime} \alpha\right)$.

Proof. By 5.5 and 5.6 we' have:

$$
\begin{aligned}
. \mu A_{2} \leqq . \mu A^{\prime} & =\int N\left(f, A^{\prime}, y\right) d \nu y=\lim _{n \rightarrow \infty} \sum \alpha \in P_{n} . \nu_{*} f\left(A^{\prime} \alpha\right) \\
& =\lim _{n \rightarrow \infty} \sum \alpha \in P_{n} . \nu_{*} f\left(A_{2} \alpha\right) \leqq . \mu A_{2} .
\end{aligned}
$$

6.11. Corollary. If $F$ is almost complete, $\nu \in \mathscr{T}(F, f), \mu=V(F, f, \nu)$, then $\mu$ is an outer measure. 
6.12. Theorem. If $F$ is almost complete, $\nu \in \mathscr{T}(F, f), \mu=V(F, f, \nu), . \mu A$ $=. \nu_{*} f A<\infty$, then $A$ is $\mu$-measurable if and only if $* A$ is $\nu$-measurable.

Proof. If $A$ is $\mu$-measurable, by 6.10 we can take $A^{\prime} \mu$-measurable so that: $A \subset A^{\prime}, . \mu A^{\prime}=\mu A$ and ${ }_{*} f A^{\prime}$ is $\nu$-measurable. Since $A$ is $\mu$-measurable:

$$
. \nu\left(* f A^{\prime}-{ }_{*} f A\right) \leqq . \nu_{*} f\left(A^{\prime}-A\right) \leqq . \mu\left(A^{\prime}-A\right)=. \mu A^{\prime}-. \mu A=0 .
$$

The converse is given by 5.8 .

6.13. TheOREM. If $F$ is almost complete, $\nu \in \Re(F, f), \mu=V(F, f, \nu), A$ is $\mu$-measurable and, for some $B C A, . \nu_{*} f B=. \nu_{*} f A$ and $. \mu B<\infty$, then $* f A$ is $\nu$-measurable.

Proof. By 4.4 choose $C \subset B$ so that $. \mu C=. \nu_{*} f C=. \nu_{*} f A$. By 6.10 , let $C^{\prime}$ be $\mu$-measurable, $C \subset C^{\prime}, . \mu C=. \mu C^{\prime}=. \nu_{*} f C^{\prime}$, and $* f C^{\prime}$ is $\nu$-measurable. Then:

$$
\begin{aligned}
. \nu\left({ }_{*} f C^{\prime}-{ }_{*} f A\right) & \leqq .{ }_{*} f\left(C^{\prime}-A\right) \leqq . \mu\left(C^{\prime}-A\right)=. \mu C^{\prime}-{ }_{\mu}\left(C^{\prime} A\right) \\
& \leqq . \mu C^{\prime}-. \mu C=0 .
\end{aligned}
$$

Thus ${ }_{*} f C^{\prime}{ }_{*} f A$ is $\nu$-measurable and $. \nu\left({ }_{*} f C^{\prime}{ }_{*} f A\right) \geqq . \nu_{*} f C=. \nu_{*} f A$ so that ${ }_{*} f A$ is also $\nu$-measurable.

6.14. Corollary. If $F$ is almost complete; $\nu \in \mathscr{T}(F, f), \mu=V(F, f, \nu), A$ is $\mu$-measurable, and for every $B \subset A$ with $. \mu B>0$ there is a $C \subset B$ with $0<. \mu C$ $<\infty$, then $* A$ is $\nu$-measurable.

6.15. Remark. In view of 6.14 above, we next turn our attention to sets which have no subset of finite positive variational measure, and see what conditions this imposes on $f$ and $\nu$.

7. Sets having no subsets of finite positive variational measure. Throughout this section we assume that $F$ is almost complete, $\nu \in \mathfrak{N}(F, f), \mu=V(F, f, \nu)$, $T \subset \sigma F, 0<. \nu_{*} f T<\infty$, and, for every $T^{\prime} \subset T$, if $. \mu T^{\prime}<\infty$, then $. \mu T^{\prime}=0$.

Our aim is to show that $f$ and $\nu$ must satisfy the conditions stated in Theorems 7.3 and 7.4. To this end, we let $P$ be a topological mesh of $F$ such that: $P_{0}=\{\sigma F\}$ and, for each $n \in \omega$ and $\alpha \in P_{n}$, there are at most two sets $\beta_{1}, \beta_{2}$ in $P_{n+1}$ with $\beta_{1} \cup \beta_{2} \subset \alpha$. This is no added restriction on $\sigma F$ since, starting with any topological mesh $P^{\prime}$ of $F$, we can define a $P$ with the above property by regrouping the elements in $\bigcup_{n} \in \omega P_{n}^{\prime}$. We choose such a $P$ only to simplify the formulas involved in the construction of certain sets.

7.1. Lemma. There exist two sets $A$ and $B$ such that:

(1) $A B=0$ and $A, B$ are $\mu$-measurable.

(2) *f $A=* f B$ and $* f A$ is $\nu$-measurable.

(3) $. \nu_{*} f(T-(A \cup B))=0$.

(4) $. \nu_{*} f(A \alpha)=. \nu_{*} f(T A \alpha)$ and $. \nu_{*} f(B \alpha)=. \nu_{*} f(T B \alpha)$ for $\alpha \in P_{1}$.

(5) For any $T^{\prime}, \sum \alpha \in P_{1} . \nu_{*} f\left(T^{\prime} A \alpha\right)=. \nu_{*} f\left(T^{\prime} A\right)$ and $\sum \alpha \in P_{1} . \nu_{*} f\left(T^{\prime} B \alpha\right)$ $=. \nu_{*} f\left(T^{\prime} B\right)$. 
(6) If $\left.A^{*} f y\right\} \neq 0$, then for some $k \in \omega: \alpha \in P_{k}, \alpha A^{*}\{y\} \neq 0$ imply $\alpha^{*} f\{y\}$ $\subset A$; and $\alpha \in P_{k}, \alpha B^{*} f\{y\} \neq 0$ imply $\alpha^{*} f\{y\} \subset B$.

Proof. Let $M^{*}$ be $\nu$-measurable, $* f T \subset M^{*}, . \nu_{*} f T=. \nu M^{*}$. We shall define the sets $M_{\alpha}$, for $\alpha \in P_{n}, A_{n}^{*}, B_{n}^{*}$ by recursion on $n$. For $\alpha \in P_{0}$ let $M_{\alpha}=M^{*}$, $A_{0}{ }^{*}=0, B_{0}^{*}=0$. Next, if $\alpha^{\prime} \in P_{n}$, let $\alpha$ and $\beta$ be in $P_{n+1}$ with $\alpha \cup \beta \subset \alpha^{\prime}$. We choose $M_{\alpha}$ and $M_{\beta} \nu$-measurable and such that

$$
\begin{aligned}
& { }_{*} f\left(T \alpha-\cup k \in n+1\left(A_{k}^{*} \cup B_{k}^{*}\right)\right) \subset M_{\alpha} \subset\left(M_{\alpha^{\prime}}-\bigcup k \in n+1{ }_{*} f A_{k}{ }^{*}\right)_{*} f \alpha, \\
& { }_{*} f\left(T \beta-\cup k \in n+1\left(A_{k}^{*} \cup B_{k}^{*}\right)\right) \subset M_{\beta} \subset\left(M_{\alpha^{\prime}}-\bigcup k \in n+1{ }_{*} f A_{k}^{*}\right)_{*} f \beta, \\
& . \nu_{*} f\left(T \alpha-\cup k \in n+1\left(A_{k}^{*} \cup B_{k}^{*}\right)\right)=. \nu M_{\alpha}, \\
& . \nu_{*} f\left(T \beta-\cup k \in n+1\left(A_{k}^{*} \cup B_{k}^{*}\right)\right)=. \nu M_{\beta} .
\end{aligned}
$$

Then we set

$$
A_{n+1}^{\left(\alpha^{\prime}\right)}=\alpha^{*} f\left(M_{\alpha} M_{\beta}\right), \quad B_{n+1}^{\left(\alpha^{\prime}\right)}=\beta^{*} f\left(M_{\alpha} M_{\beta}\right) \quad \text { if } \alpha \beta=0
$$

and $A_{n+1}^{\left(\alpha^{\prime}\right)}=B_{n+1}^{\left(\alpha^{\prime}\right)}=0$ if $\alpha=\beta=\alpha^{\prime}$.

Finally, we let

$$
\begin{aligned}
A_{n+1}^{*} & =\bigcup \alpha^{\prime} \in P_{n} A_{n+1}^{\left(\alpha^{\prime}\right)}, & B_{n+1}^{*} & =\bigcup \alpha^{\prime} \in P_{n} B_{n+1}^{\left(\alpha^{\prime}\right)} . \\
A & =\bigcup n \in \omega A_{n}{ }^{*}, & B & =\bigcup n \in \omega B_{n}{ }^{*} .
\end{aligned}
$$

Now, we observe the following facts.

(a) ${ }_{*} f A_{n}{ }^{*}={ }_{*} f B_{n}{ }^{*}$ for all $n \in \omega$.

(b) $A_{n}^{*} \cup k \in n\left(A_{k}^{*} \cup B_{k}^{*}\right)=B_{n}^{*} \cup k \in n\left(A_{k}^{*} \cup B_{k}^{*}\right)=A_{n}{ }^{*} B_{n}^{*}=0$ for all $n \in \omega$.

(c) $A_{n}^{*}, B_{n}^{*}$ are $\mu$-measurable and ${ }_{*} f A_{n}^{*}$ is $\nu$-measurable for all $n \in \omega$.

(d) $* f\left(A_{n}^{*} \alpha\right){ }_{*} f\left(A_{n}^{*} \beta\right)={ }_{*} f\left(B_{n}^{*} \alpha\right) * f\left(B_{n}^{*} \beta\right)=0$ for all $n \in \omega$ and $\alpha, \beta \in P_{n}$, $\alpha \beta=0$.

Proof. It is trivially true for $n=0$. So, let $1 \leqq n \in \omega$. In view of the choice of $P$, we can find $\alpha^{\prime}, \beta^{\prime} \in P_{k}$ and $\gamma \in P_{k-1}$, for some $k \leqq n$, such that $\alpha \subset \alpha^{\prime}$, $\beta \subset \beta^{\prime}, \alpha^{\prime} \beta^{\prime}=0, \alpha^{\prime} \cup \beta^{\prime} \subset \gamma$. If $k=n$, then $\alpha^{\prime}=\alpha, \beta^{\prime}=\beta$ and the statement is clearly true. If $k \in n$, then ${ }_{*} f\left(B_{n}^{*} \alpha\right) \cup_{*} f\left(A_{n}^{*} \alpha\right) \subset M_{\alpha} \subset M_{\alpha^{\prime}}-_{*} f A_{k}^{(\gamma)}=M_{\alpha^{\prime}}-M_{\beta^{\prime}}$, ${ }_{*} f\left(B_{n}^{*} \beta\right) \cup \cup_{*} f\left(A_{n}^{*} \beta\right) \subset M_{\beta} \subset M_{\beta^{\prime}}$.

(e) $. \nu_{*} f\left(T A_{n}^{*} \alpha\right)=. \nu_{*} f\left(A_{n}^{*} \alpha\right)$ and $. \nu_{*} f\left(T B_{n}^{*} \alpha\right)=. \nu_{*} f\left(B_{n}^{*} \alpha\right)$ for $n \in \omega, a \in P_{j}$, $j \in n+1$.

Proof. Clearly true for $n=0$. In view of (c) and (d) we may assume $n=m+1, \alpha^{\prime} \in P_{m}, \alpha \in P_{m+1}, \beta \in P_{m+1}, \alpha \beta=0$, and $\alpha \cup \beta \subset \alpha^{\prime}$. Suppose $A_{m+1}^{*} \alpha$ $=A_{m+1}^{\left(\alpha^{\prime}\right)}=\alpha^{*} f\left(M_{\alpha} M_{\beta}\right)$. Let $T_{1}=T \alpha-\bigcup k \in m+1\left(A_{k}^{*} \cup B_{k}^{*}\right)$. Then:

$$
\begin{aligned}
. \nu_{*} f T_{1} & =. \nu\left({ }_{*} f T_{1} M_{\beta}\right)+. \nu\left({ }_{*} f T_{1}-M_{\beta}\right)=. \nu * f\left(T A_{m+1}^{*} \alpha\right)+. \nu\left({ }_{*} f T_{1}-M_{\beta}\right), \\
. \nu M_{\alpha} & =. \nu\left(M_{\alpha} M_{\beta}\right)+. \nu\left(M_{\alpha}-M_{\beta}\right) .
\end{aligned}
$$

Since ${ }_{*} f T_{1} \subset M_{\alpha}$ and $. \nu_{*} f T_{1}=. \nu M_{\alpha}$, we must have: 


$$
.^{\prime \prime *} f\left(T A_{m+1}^{*} \alpha\right)=. \nu\left(M_{\alpha} M_{\beta}\right)=. \nu f\left(A_{m+1}^{*} \alpha\right) .
$$

Similarly for the other equality.

(f) $. \nu_{*} f(A \alpha)=. \nu_{*} f(T A \alpha)$ and $. \nu_{*} f(B \alpha)=. \nu_{*} f(T B \alpha)$ for $\alpha \in P_{1}$.

Proof. Since the sets ${ }_{*} f A_{n}{ }^{*}$ are $\nu$-measurable and disjoint, $A_{0}{ }^{*}=B_{0}^{*}=0$, we get from (e):

$. \nu_{*} f(A \alpha)=\sum n \in \omega . \nu_{*} f\left(A_{n}{ }^{*} \alpha\right)=\sum n \in \omega . \nu_{*} f\left(T A_{n}^{*} \alpha\right)=. \nu_{*} f(T A \alpha)$ for $\alpha \in P_{1}$.

(g) If $T^{\prime}=T-\cup k \in n+1\left(A_{k}^{*} \cup B_{k}^{*}\right)$, then $. \nu_{*} f T^{\prime}=\sum \alpha \in P_{n} . \nu_{*} f\left(T^{\prime} \alpha\right)$.

Proof. It is clearly true for $n=0$. So let $1 \leqq n \in \omega, \alpha \beta=0, \alpha, \beta \in P_{n}$. Then for some $k \leqq n, \alpha^{\prime}, \beta^{\prime} \in P_{k}, \gamma \in P_{k-1}$ we must have $\alpha \subset \alpha^{\prime}, \beta \subset \beta^{\prime}, \alpha^{\prime} \beta^{\prime}=0$, $\alpha^{\prime} \cup \beta^{\prime} \subset \gamma$. Then: ${ }_{*} f\left(T^{\prime} \alpha\right) \subset_{*} f\left(T^{\prime} \alpha^{\prime}\right) \subset M_{\alpha^{\prime}}-M_{\beta^{\prime}}$ and ${ }_{*} f\left(T^{\prime} \beta\right) \subset_{*} f\left(T^{\prime} \beta^{\prime}\right) \subset M_{\beta^{\prime}}$. Since $M_{\alpha^{\prime}}$ and $M_{\beta^{\prime}}$ are $\nu$-measurable we have the desired result.

(h) $. \nu_{*} f(T-(A \cup B))=0$.

Proof. Let $T^{\prime}=T-(A \cup B)$. Then by part $(\mathrm{g})$ and 6.10 we see that $. \mu T^{\prime}=. \nu_{*} f T^{\prime}<\infty$. Hence, $\nu_{*} f T^{\prime}=0$.

(i) For any $T^{\prime}, \sum \alpha \in P_{1} . \nu_{*} f\left(T^{\prime} A \alpha\right)=. \nu_{*} f\left(T^{\prime} A\right)$ and $\sum \alpha \in P_{1} . \nu_{*} f\left(T^{\prime} B \alpha\right)$ $=. \nu_{*} f\left(T^{\prime} B\right)$.

Proof. From Part (d), it follows that

$$
\sum \alpha \in P_{n} . \nu_{*} f\left(T^{\prime} A_{n}^{*} \alpha\right)=. \nu_{*} f\left(T^{\prime} A_{n}^{*}\right) .
$$

Hence, since $A_{0}^{*}=B_{0}^{*}=0$, we have:

$$
\begin{aligned}
\sum \alpha & \in P_{1} . \nu_{*} f\left(T^{\prime} A \alpha\right) \\
& =\sum \alpha \in P_{1} \quad \sum n \in \omega . \nu_{*} f\left(T^{\prime} A_{n}^{*} \alpha\right)=\sum n \in \omega \quad \sum \alpha \in P_{1} . \nu_{*} f\left(T^{\prime} A_{n}{ }^{*} \alpha\right) \\
& =\sum n \in \omega . \nu_{*} f\left(T^{\prime} A_{n}{ }^{*}\right)=. \nu_{*} f\left(T^{\prime} A\right) .
\end{aligned}
$$

Similarly for $B$.

(j) If $A^{*}\{y\} \neq 0$, then for some $k \in \omega: \alpha \in P_{k}, \alpha A^{*}\{y\} \neq 0$ imply $\alpha^{*} f\{y\}$ $\subset A ;$ and $\left.\alpha \in P_{k}, \alpha B^{*} f y\right\} \neq 0$ imply $\alpha^{*} f\{y\} \subset B$.

Proof. If $A^{*}\{y\} \neq 0$, then there is one and only one $k \in \omega$ such that $A_{k}{ }^{*} f\{y\} \neq 0$. Since ${ }_{*} f A_{k}{ }^{*}={ }_{*} f B_{k}{ }^{*}$, we also have $B_{k}{ }^{*}{ }^{*}\{y\} \neq 0$. Now, let $\alpha \in P_{k}$ and $\alpha A_{k}{ }^{*}{ }^{*}\{y\} \neq 0$. Since $A_{k}^{*} \alpha=\alpha^{*} f_{*} f\left(A_{k}{ }^{*} \alpha\right)$, we have $\alpha^{*} f\{y\} \subset A_{k}^{*} \alpha \subset A$. Similarly for $B$.

7.2. Lemma. There exists a sequence $G$ such that, for every $n \in \omega, G_{n+1}$ is a refinement of $G_{n}, G_{n}$ has $2^{n}$ elements, and

(1) If $A, B \in G_{n}, A \neq B$, then $A B=0$ and $A$ is $\mu$-measurable.

(2) If $A, B \in G_{n}$, then *fA=*fB and $* f A$ is $\nu$-measurable.

(3) $. \nu_{*} f\left(T-\sigma G_{n}\right)=0$.

(4) If $A \in G_{n}, \alpha \in P_{n}$, then . $\nu_{*} f(A \alpha)=. \nu_{*} f(T A \alpha)$.

(5) If $A \in G_{n}$, then $\sum \alpha \in P_{n} . \nu_{*} f\left(T^{\prime} A \alpha\right)=. \nu_{*} f\left(T^{\prime} A\right)$, for any $T^{\prime}$.

(6) If $A \in G_{n}$ and $A^{*}\left\{\{y\} \neq 0\right.$, then for some $k \in \omega: \alpha \in P_{k}$ and $\alpha A^{*}\{y\} \neq 0$ imply $\alpha^{*} f\{y\} \subset A$. 
Proof. We define $G$ by recursion. Let $M$ be $\nu$-measurable, ${ }_{*} f T \subset M$ and $. \nu_{*} f T=. \nu M$. We set $G_{0}=\left\{{ }^{*} f M\right\}$. For any $n \in \omega$, if $G_{n}$ is defined, $A \in G_{n}$ and $\alpha \in P_{n}$, we define $C_{\alpha}(A)$ and $C_{\alpha}^{\prime}(A)$ as follows: if . $\nu_{*} f(T A \alpha)=0$, let $C_{\alpha}(A)$ $=C_{\alpha}^{\prime}(A)=0$; if $._{*} f(T A \alpha)>0$ let $C_{\alpha}(A)$ and $C_{\alpha}^{\prime}(A)$ be two sets satisfying all the conditions of Lemma 7.1 with ' $T$ ' replaced by ' $T A \alpha$ ' and ' $P$ ' replaced by ' $P$ ', where $P^{\prime}$ is the sequence defined by $P_{k}^{\prime}=\mathbf{E} \alpha^{\prime}\left(\alpha^{\prime} \in P_{n+k}\right.$ and $\left.\alpha^{\prime} \subset \alpha\right)$, and such that: $C_{\alpha}(A) \cup C_{\alpha}^{\prime}(A) \subset A c$. Then we set:

$$
D(A)=\bigcup_{\alpha} \in P_{n} C_{\alpha}(A) \text { and } D^{\prime}(A)=\bigcup_{\alpha} \in P_{n} C_{\alpha}^{\prime}(A) .
$$

We note that, since

$$
. \nu_{*} f T=. \nu_{*} f\left(T \sigma G_{n}\right) \leqq . \nu_{*} f\left(\sigma G_{n}\right)=. \nu_{*} f A=. \nu_{*} f(T A) \leqq . \nu_{*} f T,
$$

we must have for some $\alpha \in P_{n}, . \nu_{*} f(T A \alpha)>0$. Thus, $D(A) \neq D^{\prime}(A)$ and they both satisfy all conditions of 7.1 with ' $P_{1}$ ' replaced by ' $P_{n+1}$ ' in (4) and (5). Finally, to insure property (2), we note that . $\nu_{*} f A=. \nu_{*} f(T A)=. \nu_{*} f(T D(A))$ $=. \nu_{*} f(D(A))$ so that the sets

$$
\begin{aligned}
\bar{D}(A) & =D(A)-\cup B \in G_{n}{ }^{*} f\left({ }_{*} f A-{ }_{*} f(D(B))\right), \\
\bar{D}^{\prime}(A) & =D^{\prime}(A)-\cup B \in G_{n}{ }^{*} f\left({ }_{*} f A-{ }_{*} f(D(B))\right)
\end{aligned}
$$

differ from $D(A)$ and $D^{\prime}(A)$ respectively by a set of $\mu$-measure zero, and ${ }_{*} f(\bar{D}(A))={ }_{*} f(\bar{D}(B))$ for $B \in G_{n}$. Therefore, we let

$$
G_{n+1}=\bigcup A \in G_{n}\left(\{\bar{D}(A)\} \cup\left\{\bar{D}^{\prime}(A)\right\}\right)
$$

and see that properties (1) through (6) are satisfied with ' $n$ ' replaced by ' $n+1$ '.

7.3. Theorem. If $F$ is almost complete, $\nu \in \mathfrak{M}(F, f), \mu=V(F, f, \nu), T \subset \sigma F$, $0<. \nu_{*} f T<\infty$, and, for every $A \subset T, . \mu A<\infty$ implies . $\mu A=0$, then there exists a sequence $S$ such that, for every $n \in \omega, S_{n+1}$ is a refinement of $S_{n}$ and

(1) $\sigma S_{n}=\sigma S_{0} \subset * f T$,

(2) $A \in S_{n}, B \in S_{n}, A \neq B$ imply $A B=0$,

(3) $A \in S_{n}$ implies . $\nu A=. \nu_{*} T T$,

(4) $B \subset \sigma S_{0}$ and $. \nu B \neq 0$ imply $\lim _{n \rightarrow \infty} \sum A \in S_{n} . \nu(B A)=\infty$.

Proof. Let $T^{\prime} \subset T$ be such that ${ }_{*} f T^{\prime}={ }_{*} f T$ and $f(x) \neq f(y)$ whenever $x \in T^{\prime}$, $y \in T^{\prime}, x \neq y$. Let $G$ be a sequence satisfying the conditions in 7.2 with ' $T$ ' replaced by ' $T$ '. Let $T_{0}={ }_{*} f \cap n \in \omega\left(T^{\prime} \sigma G_{n}\right)$. We define $S$ by setting

$$
S_{n}=\cup A \in G_{n}\left\{T_{0} * f\left(T^{\prime} A\right)\right\} \quad \text { for } n \in \omega
$$

Properties (1) and (2) are clearly satisfied. Also

$$
. \nu\left({ }_{*} f T^{\prime}-T_{0}\right) \leqq \sum n \in \omega . \nu_{*} f\left(T^{\prime}-\sigma G_{n}\right)=0 .
$$

Hence: 


$$
. \nu\left(T_{0 *} f\left(T^{\prime} A\right)\right)=. \nu_{*} f\left(T^{\prime} A\right)=. \nu_{*} f T^{\prime} \quad \text { for } A \in G_{n} .
$$

Finally, if $B \subset T_{0}$ and $. \nu B \neq 0$, let $C=\bigcap n \in \omega\left(T^{\prime} \sigma G_{n} *_{f} B\right)$. Then:

$$
\begin{aligned}
\infty & =\lim _{n \rightarrow \infty} \sum \alpha \in P_{n} . \nu_{*} f(C \alpha) \leqq \lim _{n \rightarrow \infty} \sum \alpha \in P_{n} \quad \sum A \in G_{n} . \nu_{*} f(C A \alpha) \\
& =\lim _{n \rightarrow \infty} \sum A \in G_{n} . \nu_{*} f(C A)=\lim _{n \rightarrow \infty} \sum A \in G_{n} . \nu\left({ }_{*} f C_{*} f\left(T^{\prime} A\right)\right) \\
& =\lim _{n \rightarrow \infty} \sum A \in S_{n} . \nu(B A) .
\end{aligned}
$$

7.4. ThEOREM. If $F$ is almost complete, $\nu \in \mathfrak{M}(F, f), \mu=V(F, f, \nu), T \subset \sigma F$, $0<. \nu_{*} f T<\infty$, and, for every $A \subset T, . \mu A<\infty$ implies $. \mu A=0$, then there exists $T^{*}, \nu$-measurable and . $\nu T^{*}>0$, such that, for every $y \in T^{*},{ }^{*}\{y\}$ is noncountable.

Proof. Let $G$ be a sequence satisfying 7.2 $T^{\prime}=\cap n \in \omega * f \sigma G_{n}$, and $T^{*}=\mathbf{E} y$ ( $y \in T^{\prime}$ and ${ }^{*} f\{y\}$ is closed). Clearly $T^{*}$ is $\nu$-measurable and $. \nu T^{*}=. \nu T^{\prime}$ $=. \nu_{*} f T>0$.

Let $y \in T^{*}$ and $S=\mathrm{E} A$ ( $A$ is a sequence and $A_{n+1} \subset A_{n} \in G_{n}$ for all $n \in \omega$ ). If $A \in S$, we have $A_{n}{ }^{*}\{y\} \neq 0$ for all $n \in \omega$, and hence by 6.5 there is an $\alpha \in S q(P)$ such that $\alpha_{n} A_{n}{ }^{*}\{y\} \neq 0$. By property (6) in 7.2 , this means $0 \neq \alpha_{n+k}{ }^{*}\{y\} \subset A_{n}$ for some $k \in \omega$. Hence if $x \in \cap n \in \omega \alpha_{n}$, then $x$ is in the closure of ${ }^{*} f\{y\}$, therefore $x \in{ }^{*} f\{y\}$ and $x \in \cap n \in \omega A_{n}$. If $B \in S, B \neq A$, and $\alpha^{\prime} \in S q(P), \alpha_{n}^{\prime} B_{n}{ }^{*}\{y\} \neq 0$ for all $n \in \omega$, then $\alpha^{\prime} \neq \alpha$. Since there are a countable number of $\alpha \in S q(P)$ such that $\cap n \in \omega a_{n}=0$, and $S$ is noncountable, it follows that $S^{\prime}=E A\left(A \in S\right.$ and $\left.T^{*}={ }_{*} f \cap n \in \omega A_{n}\right)$ is noncountable, and since $A \in S^{\prime}, B \in S^{\prime}, A \neq B$ implies $A_{n} B_{n}=0$ for some $n \in \omega$, this means ${ }^{*}\{y\}$ is noncountable for all $y \in T^{*}$.

7.5. Remark. Since $F$ has a topological mesh, there can be at most a countable number of disjoint neighborhoods in $\sigma F$. From this it follows that any closed subset of $\sigma F$ is noncountable if and only if it contains a nontrivial perfect subset. The fact that, in $7.4,{ }^{*} f\{y\}$ contains a nontrivial perfect subset for all $y \in T^{*}$ can also be seen directly by making a small change in the proof.

8. Applications. In this section we indicate some immediate consequences of the theory developed so far when it is applied to functions on the real line. Extensions to other spaces can easily be seen.

8.1. Definition. $\langle a, b\rangle=\mathbf{E} t(t$ is irrational and $a\langle t\rangle b)$.

8.2. Definition. $F_{0}=\mathrm{E} \alpha(\alpha=\langle a, b\rangle$ for some rational $a, b$ with $0 \leqq a<b$ $\leqq 1)$.

8.3. Definition. $\mathfrak{T}_{0}=\mathbf{E} \nu$ ( $\nu$ is an outer measure on $[0,1]$ and intervals are $\boldsymbol{\nu}$-measurable).

8.4. Definition. $\mathfrak{T}_{1}=\mathrm{E} \nu\left(\nu \in \mathbb{T}_{0} ; . \nu[0,1]<\infty ; . \nu\{y\}=0\right.$ for $\left.0 \leqq y \leqq 1\right)$.

8.5. Definition. $\mathfrak{T}_{2}=\mathrm{E} \nu\left(\nu \in \mathfrak{T}_{0}\right.$; there exists no sequence $S$ such that, 
for $n \in \omega: S_{n+1}$ is a refinement of $S_{n} ; \sigma \cdot S_{n}=\sigma S_{0} \subset[0,1] ; A \in S_{n}, B \in S_{n}, A \neq B$ imply $A B=0 ; 0<. \nu \sigma S_{0}<\infty ; B \subset \sigma S_{0}$ and $. \nu B \neq 0$ imply $\lim _{n \rightarrow \infty} \sum A \in S_{n}$ $. \nu(A B)=\infty)$.

8.6. Definition. $A$ is measurable $M$ if and only if $A$ is $\nu$-measurable for all $\nu \in M$.

8.7. Definition. $A$ has absolutely $M$ measure zero if and only if $. \nu A=0$ for all $\nu \in M$.

8.8. Definition. $\mathcal{F}(M)=\mathrm{E} f$ ( $f$ is a function on $\sigma F_{0}$ to $[0,1] ; * f \alpha$ is measurable $M$ for all $\alpha \in F_{0} ;{ }^{*}\{y\}$ is closed for all $y \in \operatorname{rng} f-Z$ where $Z$ has absolutely $M$ measure zero).

We observe that: $\sigma F_{0}$ is the set of irrationals in $[0,1]$, and $F_{0}$ is almost complete; $A$ is measurable $\mathfrak{T}_{0}$ if and only if $A$ is measurable $\mathfrak{T}_{1}$; if $f \in \mathcal{F}\left(\mathfrak{T}_{1}\right)$ and $\nu \in \mathscr{M}_{1}$ then $\nu \in \mathscr{M}\left(F_{0}, f\right)$ and $\mu=V\left(F_{0}, f, \nu\right) \in \mathscr{M}_{0}$ (if we extend $\mu$ to all of $[0,1]$ by setting . $\mu A=0$ for all sets $A$ of rationals); if $f \in \mathscr{F}\left(\mathfrak{N}_{2}\right)$ and $\nu \in \mathfrak{N}_{2}$ then $\nu \in \mathscr{M}\left(F_{0}, f\right)$ and $V\left(F_{0}, f, \nu\right) \in \mathfrak{M}_{2}$. Then, using $6.13,7.3$ and 7.4 we conclude:

8.9. Theorem. If $f \in \mathcal{F}\left(\mathfrak{T}_{2}\right)$ and $A$ is measurable $\mathfrak{T}_{2}$ then ${ }_{*} f A$ is measurable $\mathfrak{T l}_{2}$.

8.10. Theorem. If $f \in \mathcal{F}\left(\mathfrak{M}_{1}\right)$ and ${ }^{*}\{y\}$ is countable for $y \in \operatorname{rng} f-Z$, for some $Z$ of absolutely $\mathfrak{M}_{1}$ measure zero, and $A$ is measurable $\mathfrak{M}_{0}$, then ${ }_{*} f A$ is measurable $\mathfrak{M}_{0}$.

8.11. Corollary. If $f$ is continuous on the irrationals to $[0,1]$ and $A$ is measurable $\mathfrak{T C}_{2}$, then ${ }_{*} f A$ is measurable $\mathfrak{M}_{2}$.

Proof. $* f \alpha$ is analytic for $\alpha \in F_{0}$. Thus $f \in \mathcal{F}\left(\mathscr{T}_{0}\right)$.

8.12. Corollary. If $f$ is continuous on the irrationals to $[0,1],{ }^{*}\{y\}$ is countable for $y \in \operatorname{rng} f-Z$, for some $Z$ of absolutely $\mathfrak{M C}_{1}$ measure zero, and $A$ is measurable $\mathfrak{M}_{0}$, then ${ }_{*} f A$ is measurable $\mathfrak{T}_{0}$.

8.13. Remark. A set $Z$ of absolutely $\mathfrak{T}_{1}$ measure zero need not be countable (see [1]).

8.14. Remark. An immediate consequence of 8.11 is that all projective sets are measurable $\mathfrak{T}_{2}$. Since K. Gödel has indicated (see [2]) that the existence of a projective set $P_{2}$ which is not Lebesgue-measurable is consistent with the usual axioms of set theory if the latter are consistent, it follows that we cannot have Lebesgue measure in $\mathfrak{M}_{2}$. It would be interesting to show directly that Lebesgue measure is not in $\mathfrak{T H}_{2}$, i.e., if one could produce a sequence satisfying conditions (1) through (4) in Theorem 7.3 with $\nu$ taken to be Lebesgue measure.

On the other hand, M. Kondô (see [4]) has shown that for every projective set $P_{2}$ of class 2 (see [6] for general definitions) there is a set $A$, whose complement is analytic, and a function $f$, continuous and one-to-one on $A$, 
such that ${ }_{*} f A=P_{2}$. It is well known that $f$ can be extended to a $G_{\delta}$ containing $A$, with continuity preserved. In view of $\mathrm{K}$. Gödel's result mentioned above and 8.12, it follows that such an extension cannot always assume every value $y$ only a countable number of times.

It may also be of interest to note that the results of this section may be extended immediately to complete, separable metric spaces since any such space that is also noncountable is the image within a countable set, of the set of irrationals by a one-to-one continuous function.

\section{BIBLIOGRAPHY}

1. A. S. Besicovitch, Concentrated and rarified sets of points, Acta Math. vol. 62 (1934) pp. 289-300.

2. K. Gödel, The consistency of the axiom of choice and of the generalized continuum-hypothesis, Proc. Nat. Acad. Sci. U.S.A. vol. 24 (1938) pp. 556-557.

3. H. Hahn and A. Rosenthal, Set functions, 1948.

4. M. Kondô, Sur l'uniformisation des complémentaires analytiques et les ensembles projectifs de la seconde classe, Japanese Journal of Mathematics vol. 15 (1939) pp. 197-230.

5. M. Lavrentieff, Contribution d la théorie des ensembles homéomorphes, Fund. Math. vol. 6 (1924) pp. 149-160.

6. N. Lusin, Leçons sur les ensembles analytiques et leurs applications, 1930.

7. M. E. Munroe, Introduction to measure and integration, 1953.

8. M. W. Sierpinski, Les ensembles projectifs et analytiques, Mémorial des Sciences Mathematiques, no. $112,1950$.

The Institute for Advanced Study, Princeton, N. J. 